\title{
Compensatory growth responses to food restriction in the Chinese three-keeled pond turtle, Chinemys reevesii
}

\author{
Chunxia Xu, Wei Xu and Hongliang Lu*
}

\begin{abstract}
Juvenile Chinese three-keeled pond turtles (Chinemys reevesii) were subjected to one of four different feeding regimens: ad libitum (AL), restricted (R), ad libitum-restricted (AL-R), or restricted-ad libitum (R-AL) for 13 weeks, to assess the compensatory growth (CG) response to food restriction and subsequent re-alimentation. After switching to ad libitum feeding, the turtles in R-AL group ate more food and grew faster than those in other groups. At the end of the trial, R-AL turtles achieved the comparable body weight as AL turtles, indicating that a complete CG response occurred. Cumulative food consumption over the entire period did not differ between R-AL turtles and AL turtles. Experimental treatment affected carcass composition. Carcass lipid content of AL turtles was greater than that of R and AL-R turtles, with R-AL turtles in between. Carcass protein content of R-AL turtles was slightly greater than that of other groups without statistical differences. Stored lipids might be consumed firstly when animals underwent food restriction. Our results reconfirmed the CG of C. reevesii after food restriction. However, it is still difficult to achieve a reduction in the cost of farm-raised turtle production by adopting a restricted-satiation feeding protocol.
\end{abstract}

Keywords: Chinemys reevesii; Compensatory growth; Food consumption; Carcass composition

\section{Background}

For many centuries, turtles have been used as food, pets and in traditional medicine in different regions of the world (Fordhama et al. 2007; Mutalib et al. 2013). However, long-term over-exploitation of wild turtles is currently threatening their survival (Fong et al. 2007; Buhlmann et al. 2009). In the past few decades, a number of turtle species have been artificially cultured in an effort to satisfy the increasing demand for turtles in countries such as China. In commercial aquaculture, greater growth rate means a shortening of the culture cycle of farm-raised animals, of which could effectively reduce food consumption and production costs, therefore, finding the proper husbandry strategies to increase the growth rates of aquatic animals is very critical for farmers.

Compensatory growth (CG), the phase of accelerated growth following a period of feed restriction, has been observed in various organisms from mollusks to mammals

\footnotetext{
* Correspondence: honglianglu@live.cn

Hangzhou Key Laboratory for Animal Adaptation and Evolution, School of Life and Environmental Sciences, Hangzhou Normal University, Hangzhou 310036, People's Republic of China
}

(Wilson and Osbourn 1960; Fermin 2002; Vonesh and Bolker 2005; Wei et al. 2008; Roark et al. 2009). In many cases, food-restricted or food-deprived animals can eventually achieve the same or even a greater body size upon return to favorable food conditions, compared with those that have not experienced food restriction (Ali et al. 2003; Jobling 2010; Won and Borski 2013). Accordingly, it may be possible to exploit the principle of CG to improve the growth rates of farm-raised animals. This has previously been demonstrated in certain fish species (Jobling et al. 1994; Hayward et al. 1997; Chatakondi and Yant 2001). For example, juvenile hybrid sunfish (Lepomis macrochirus $\times L$. gibbosus) that undergo repeating cycles of deprivation and re-feeding grow significantly faster and achieve a greater size at the same age than controls that are fed to satiation daily (Hayward et al. 1997). Among cultured aquatic species, studies addressing CG have focused mainly on fish (e.g., Hayward et al. 2000; Oh and Noh 2006; Srijila et al. 2014). Although an evident compensatory response to food deprivation has been found in 
some cultured freshwater turtles (Xie and Niu 2007; Wang et al. 2011; Xie et al. 2012), it remains unclear whether the growth rate of these turtles can be improved by exploiting the CG response.

The Chinese three-keeled pond turtle, Chinemys reeve$s i i$, is a species that is widely distributed in eastern Asia from Japan to southern China. This species is one of the most commercially important turtles for aquaculture and is widely cultured in China (Cheung and Dudgeon 2006; Du et al. 2007). A CG response to complete food deprivation in $C$. reevesii was demonstrated in a previous study, in which deprived turtles were refed to satiation for 4 weeks, but did not achieve the same size as controls (Wang et al. 2011). However, the magnitude of compensatory growth may depend on the developmental stage of the animals, and the intensity and duration of feed restriction (Ali et al. 2003). Seasonal fluctuations in food availability are ubiquitous in the natural environment and wild animals often undergo intermittent, partial food deprivation rather than prolonged, complete food deprivation. In fact, the growth responses of cultured turtles to limited food availability remain largely unstudied. More detailed information is necessary in order to determine whether CG can be used to improve the growth rate of $C$. reevesii. In the present study, we assessed the compensatory responses of juvenile C. reevesii to food restriction followed by increased food availability, thereby providing useful information for turtle husbandry practices.

\section{Materials and methods}

\section{Animal collection and maintenance}

A total of 62 juvenile turtles, about 2 months after hatching, were obtained from a private hatchery in Haining (Zhejiang, eastern China), and transferred to our laboratory at Hangzhou Normal University, where they were weighed and measured for carapace length and width. The turtles were housed individually in $30 \times$ $20 \times 25 \mathrm{~cm}^{3}$ aquaria that contained water to a depth of $5 \mathrm{~cm}$. Aquaria were kept in a temperature-controlled room at $30^{\circ} \mathrm{C}$ under a $12 \mathrm{~h}$ light: $12 \mathrm{~h}$ dark cycle. Pieces of tiles were placed in the aquaria to provide shelters for the turtles, and the water was replaced daily. Turtles were fed a commercially available diet (food composition: 10\% water, $47 \%$ crude proteins, $8 \%$ lipids and $7 \%$ carbohydrates) daily, and the food pellets that remained in each aquarium were counted every afternoon. Approximate food consumption was calculated as the difference between the mass of food offered and the estimated mass of food remaining (the number of remaining food pellets $x$ the average mass per pellet). Turtles were weighed weekly.

After 2 weeks of acclimation to the laboratory, turtles were randomly assigned to one of four treatment groups following Roark et al. (2009): ad libitum (AL; fed ad libitum for 13 weeks, $N=13$ ), restricted (R; fed $\sim 25 \%$ of initial ad libitum intake for 13 weeks, $N=13$ ), ad libitum-restricted (AL-R; fed ad libitum for 6 weeks and then food-restricted for 7 weeks, $N=13$ ), and restrictedad libitum (R-AL; food-restricted for 6 weeks and then fed ad libitum for 7 weeks, $N=13$ ). The remaining turtles $(N=10)$ were killed and hereafter are referred to as 0 -week turtles.

\section{Carcass composition}

After 13 weeks, all turtles were euthanized by freezing to $-15^{\circ} \mathrm{C}$ for later determination of composition. Each turtle was separated into the carcass (including head, limbs, tail, carapace and plastron) and internal organs. The carcasses were dried to constant mass in an oven at $65^{\circ} \mathrm{C}$, and then weighed to the nearest $0.1 \mathrm{mg}$ on a Mettler Toledo balance (model AB135-S). The whole dried carcass was ground in a Wiley mill to a fine powder for subsequent analyses of the lipid and protein content. We extracted non-polar lipids from dried carcass samples in a Soxhlet apparatus for $5.5 \mathrm{~h}$ using absolute ether as solvent. The lipid content of each sample was determined by subtracting the lipid-free dry mass from the total sample dry mass. Nitrogen content was determined by the Kjeldahl method (AOAC 1984), and protein content was calculated by multiplying nitrogen content by 6.25 .

\section{Data analysis}

One turtle in R-AL group died during the experiment and the corresponding data were excluded from statistical analysis. The specific growth rate (SGR) and feed efficiency ratio (FER) were respectively calculated as SGR = $\left(\ln W_{\mathrm{t}}-\ln W_{0}\right) / T \times 100 \%$ and FER $=\left(W_{\mathrm{t}}-W_{0}\right) / C_{\mathrm{w}} \times 100 \%$, where $W_{0}=$ initial wet body mass, $W_{\mathrm{t}}=$ final wet body mass, $T=$ duration of experiment and $C_{\mathrm{w}}=$ wet mass of food consumed. Statistical analyses were performed using STATISTICA 6.0 (StatSoft Inc. OK, USA). We used linear regression, one-way analysis of variance (ANOVA), repeated measures ANOVA, multivariate analysis of variance (MANOVA) and Tukey's post hoc test to analyze the corresponding data that met the assumptions for parametric analyses. Before conducting parametric analyses, all variables were tested for normality using the KolmogorovSmirnov test and for homogeneity of variances using Bartlett's (at univariate level) or Box's M (at multivariate level) test. Throughout the paper, values are presented as mean $\pm \mathrm{SE}$, and the significance level is set at $P=0.05$.

\section{Results}

The body size (mass) of turtles did not differ among groups at week 0 (prior to the beginning of the experiment) (one-way ANOVA, $F_{4,56}=0.14, P=0.966$ ), but significantly differed at week 6 (prior to the diet switch) $\left(F_{3,47}=3.39, P=0.026\right)$ and at week 13 (the end of the 
experiment $)\left(F_{3,47}=7.72, P<0.001\right)$. Repeated measures ANOVA revealed significant effects of time $\left(F_{13,611}=\right.$ 172.70, $P<0.0001)$, treatment $\left(F_{3,47}=3.09, P=0.036\right)$, and a time $\times$ treatment interaction $\left(F_{39,611}=12.05, P<0.0001\right)$ on body size throughout the trial. The mean body size of R-AL and R turtles was significantly smaller than that of $\mathrm{AL}$ and AL-R turtles at week 6. After ad libitum feeding for 7 weeks, mean body size of R-AL turtles was similar to that of AL turtles, and was significantly larger than that of $\mathrm{R}$ turtles (Figure 1).

The potential influences of body size on the growth rate, food consumption and FER of the turtles were not found among treatments (linear regression, all $P>0.05$ ). Foodrestricted turtles were had significantly lower food consumption (one-way ANOVA, $F_{3}, \quad 47=128.09$, $P<0.0001, \mathrm{AL}-\mathrm{R}=\mathrm{AL}>\mathrm{R}=\mathrm{AL}-\mathrm{R})$ and SGR $\left(F_{3,47}=6.09\right.$, $P<0.002, \mathrm{AL}=\mathrm{AL}-\mathrm{R}>\mathrm{R}=\mathrm{R}-\mathrm{AL})$, but higher FER $\left(F_{3,47}=\right.$ 2.86, $P=0.047)$ than ad libitum-fed turtles during weeks 0-6 (Figure 2). R-AL turtles consumed significantly more food (repeated measures ANOVA, $F_{1,}, 11=884.16$, $P<0.0001)$ and exhibited a higher growth rate $\left(F_{1,11}=\right.$ 89.41, $P<0.0001)$ after the switch to ad libitum feeding, whereas AL-R turtles exhibited a lower growth rate $\left(F_{1,12}=9.86, P<0.01\right)$ due to restricted feeding $\left(F_{1,12}=\right.$ 1028.77, $P<0.0001$ ). Food consumption (one-way ANOVA, $F_{3,47}=122.97, P<0.0001, \mathrm{R}-\mathrm{AL}>\mathrm{AL}>\mathrm{R}=$ AL-R) and SGR $\left(F_{3,47}=27.76, P<0.0001, \mathrm{R}-\mathrm{AL}>\mathrm{AL}>\right.$ $\mathrm{R}=\mathrm{AL}-\mathrm{R})$ also differed between groups during weeks 7-13, with R-AL turtles having a greater food intake and growing faster than turtles in other groups (Figure 2). Over the 13-week period, the cumulative food consumption of R-AL turtles was similar to that of AL turtles, but was significantly higher than those of AL-R and R turtles (one-way ANOVA, $F_{3,47}=112.25, P<0.0001$, $\mathrm{AL}=\mathrm{R}-\mathrm{AL}>\mathrm{AL}-\mathrm{R}=\mathrm{R})$. In order to assess CG capacity, we excluded the data from AL-R and R turtles, and compared R-AL turtles to AL turtles in weeks 7-13. After the switch to ad libitum feeding, the SGR of R-AL turtles was higher than that of AL turtles in the first few weeks, and gradually returned to the level of AL turtles in the last 3 weeks (Figure 3). The FER of turtles did not differ among groups during weeks 7-13 (one-way ANOVA, $F_{3,47}=1.83, P=0.154$ ).

Experimental treatment affected carcass composition (MANOVA, Wilks' $\lambda=0.44, d f=15,119, P<0.01$ ). Carcass wet and dry masses of AL and R-AL turtles were greater than that of $\mathrm{R}$ turtles. The water content of wet carcass did not differ among groups. The lipid content of dry carcass of AL turtles was greater than those of R and AL-R turtles, but did not differ from that of R-AL turtles. The protein content of R-AL was slightly greater than that of other groups, but the difference was not statistically significant (Table 1). Carcass wet and dry masses of 0 -week turtles were significantly smaller than those of the experimental turtles. The lipid content of 0 -week turtles was greater than those of AL-R and $\mathrm{R}$ turtles, but did not differ from those of AL and R-AL turtles (Table 1).

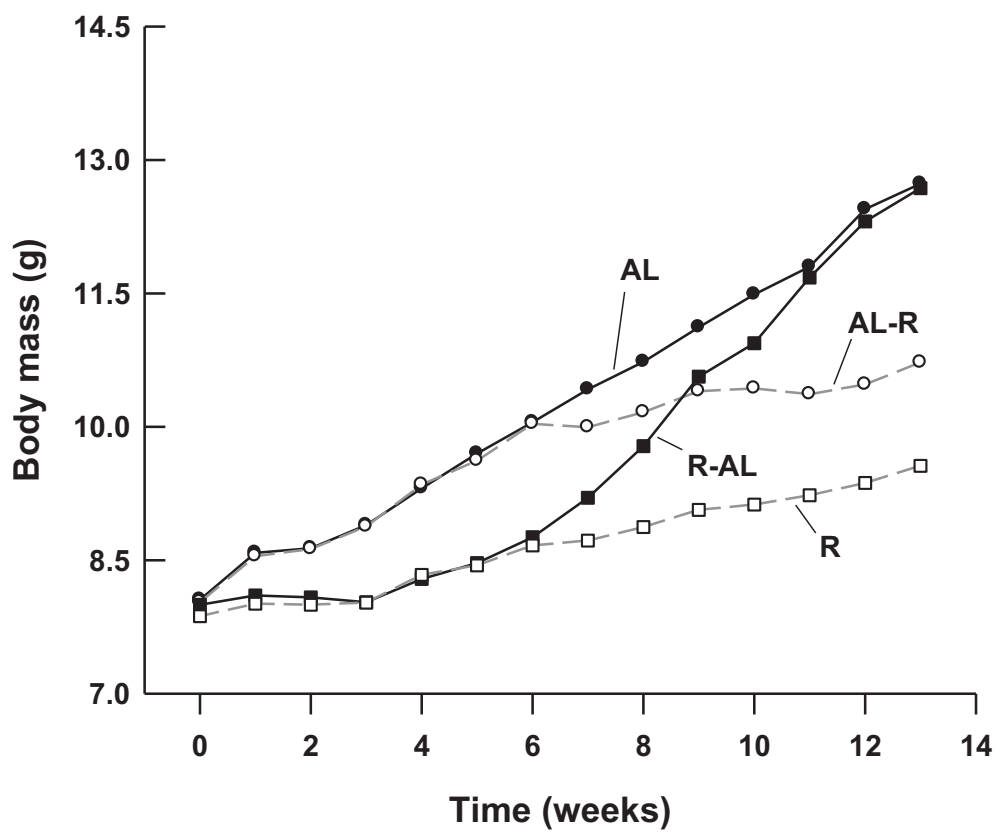

Figure 1 Mean body mass of juvenile Chinemys reevesii fed ad libitum (AL), restricted (R), ad libitum-restricted (AL-R), or restricted-ad libitum (R-AL) diets. Body mass was measured th the end of each week. 

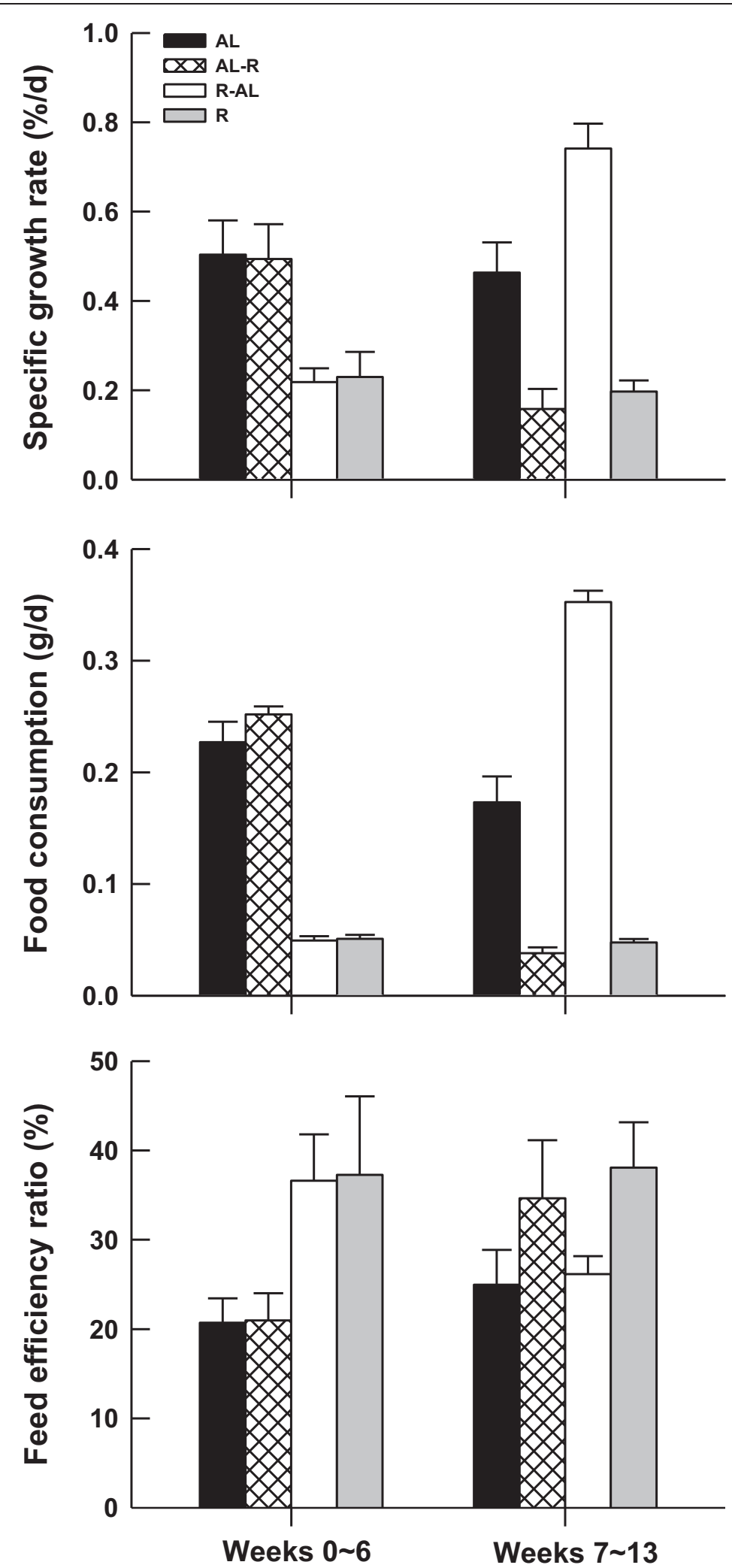

Figure 2 Specific growth rate, food consumption, and feed efficiency ratio for juvenile Chinemys reevesii fed ad libitum (AL), restricted (R), ad libitum-restricted (AL-R), or restricted-ad libitum (R-AL) diets. Data are expressed as mean + SE. 


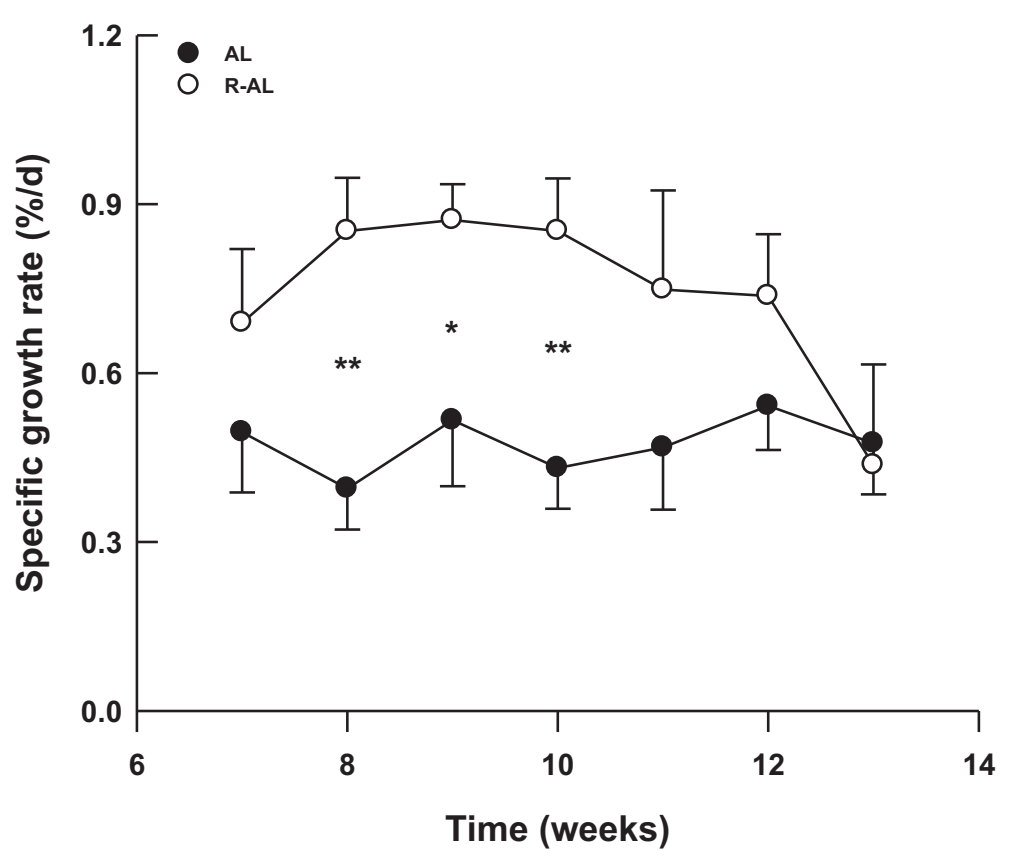

Figure 3 Specific growth rate of Chinemys reevesii fed ad libitum (AL; mean - SE) and restricted-ad libitum (R-AL; mean + SE) diets in weeks 7-13. The asterisks denote significant differences in specific growth rate between AL and R-AL turtles. ${ }^{*} P<0.05$, ${ }^{* *} P<0.01$.

\section{Discussion}

Consistent with the results of Wang et al. (2011), an obvious CG response was observed in juvenile C. reevesii that experienced a period of feed restriction. Foodrestricted turtles caught up in body size with those that had not undergone feed restriction, indicating a complete CG pattern in C. reevesii. However, Wang et al. (2011) reported a partial CG response in this turtle. The expression of CG responses can be affected by several factors, such as the size of the animals, the feeding regime, and the intensity and duration of feed restriction (Kim and Lovell 1995; Thompson et al. 2000; Ali et al. 2003). The differences between the results of these two studies may be due to differences in the experimental methods or the size of animals. Turtles in the Wang et al. (2011) study had relatively large size and were completely deprived of food for no more than 4 consecutive weeks, whereas R-AL turtles in the present study were fed a restricted ration for 6 weeks. The deprivation period should be long enough (more than 2 weeks) to evoke a detectable CG response, and a longer deprivation period seems to result in a more pronounced response in C. reevesii (Wang et al. 2011). The complete CG response we observed was probably a result of the relatively long period of food restriction. Therefore, the results in our study may not contradict those reported by Wang et al. (2011), but provide evidence that the intensity and duration of feed restriction affects the CG response of juvenile turtles. Moreover, the influence of feed restriction intensity on CG response may vary among species or populations. For example, restricted feeding induces an obvious CG response in C. reevesii, which has also been observed in two sea turtles, Caretta caretta and Chelonia mydas (Bjorndal et al. 2003; Roark et al. 2009). However, another freshwater turtle, Pelodiscus sinensis, exhibited only a partial CG response to complete food deprivation (Xie and Niu 2007).

CG can be attributed to starvation-induced physiological changes (Ali et al. 2003; Gurney et al. 2003; Won

Table 1 Carcass composition of juvenile Chinemys reevesii at 0 - and 13 -weeks

\begin{tabular}{lllllll}
\hline Group & $\boldsymbol{N}$ & Body wet mass (g) & Dry mass $(\mathbf{g})$ & Water content (\%) & Lipid content (\%) & Protein content (\%) \\
\hline 0-week turtles & 10 & $5.46 \pm 0.18^{\mathrm{c}}$ & $1.31 \pm 0.05^{\mathrm{c}}$ & $75.7 \pm 1.4$ & $11.2 \pm 0.6^{\mathrm{a}}$ & $7.8 \pm 0.6$ \\
$\mathrm{AL}$ & 13 & $9.82 \pm 0.56^{\mathrm{a}}$ & $2.39 \pm 0.12^{\mathrm{a}}$ & $75.5 \pm 0.5$ & $10.5 \pm 0.6^{\mathrm{a}}$ & $7.6 \pm 0.4$ \\
$\mathrm{AL}-\mathrm{R}$ & 13 & $8.37 \pm 0.54^{\mathrm{ab}}$ & $1.86 \pm 0.22^{\mathrm{bc}}$ & $77.5 \pm 2.7$ & $8.2 \pm 0.7^{\mathrm{b}}$ & $8.2 \pm 0.6$ \\
R-AL & 12 & $9.70 \pm 0.35^{\mathrm{a}}$ & $2.22 \pm 0.13^{\mathrm{ab}}$ & $77.1 \pm 1.1$ & $9.9 \pm 0.7^{\mathrm{ab}}$ & $8.7 \pm 0.3$ \\
R & 13 & $7.36 \pm 0.18^{\mathrm{b}}$ & $1.68 \pm 0.04^{\mathrm{c}}$ & $77.1 \pm 0.3$ & $8.0 \pm 0.5^{\mathrm{b}}$ & $7.0 \pm 0.4$ \\
\hline
\end{tabular}

Data are expressed as mean \pm SE. Means with different lettered superscripts differ significantly (Tukey's post hoc test, $a>b>c$ ). 
and Borski 2013). After the switch to ad libitum feeding, food consumption of R-AL turtles were significantly enhanced and greater than that of AL turtles, whereas the FER in R-AL turtles was lower than other groups without statistical differences. This suggests that an increase in food consumption (i.e., hyperphagia), rather than the efficiency of food conversion, may be the major cause for CG in C. reevesii. Enhancement of growth rates by increasing food consumption was also found in the previous study (Wang et al. 2011), and in P. sinensis (Xie and Niu 2007) and some fish species (Chatakondi and Yant 2001; Tian and Qin 2004). CG may also be achieved by improving food conversion and reducing metabolic rate (Ali et al. 2003; Roark et al. 2009). Completely food-deprived $C$. reevesii juveniles have lower metabolic rates than those fed to satiation (Lu and Wang 1993). The reduced metabolic rate of deprived turtles might persist for the first few days of refeeding period, and contributes to the CG response (Wang et al. 2011). The restricted ration used in this study may have exceeded basal maintenance costs because food-restricted turtles continued to grow during the restriction period. Whether the metabolic rate of food-restricted turtles is reduced during the periods of restriction and ad libitum feeding should be determined in future research.

The lipid content of dry carcasses appears to be related to the duration of feed restriction, with turtles that experienced a longer period of food restriction having lower carcass lipid content. However, R-AL turtles tended to have relatively high carcass protein content at the end of the experiment (Table 1). Our results suggest that stored lipids may be mobilized prior to the mobilization of proteins when animals undergo food restriction or deprivation. Such pattern was also exhibited in the previous study (Wang et al. 2011), and in P. sinensis (Xie and Niu 2007) and some fish species (Qian et al. 2000; Luo et al. 2009).

In summary, the present study confirmed that juvenile three-keeled pond turtles exhibit a complete CG response following a period of food restriction. However, the R-AL feeding regime that we used did not reduce the total food consumption of turtles over the entire experiment. Accordingly, this protocol is not recommended for aquaculture practices, because it fails to evoke an overcompensation of growth, and thus shorten the duration of culture cycles or reduce food consumption of farm-raised turtles.

\section{Competing interests}

The authors declare that they have no competing interests.

\section{Authors' contributions}

CXu and WXu carried out the experiments and participated in data analysis. HLu conceived of the study and participated in its design. CXU and HLu wrote the final version of the manuscript. All authors read and approved the final version of the manuscript.

\section{Acknowledgments}

The work complied with the current laws on animal welfare and research in China, and was supported by grants from the National Science Foundation of Zhejiang Province (Y3110276), Zhejiang Provincial Public Technology Application Research Project (2012C22060) and Scientific Research Program of Hangzhou City (20110332H04)

Received: 5 September 2014 Accepted: 13 November 2014 Published: 24 November 2014

\section{References}

Ali M, Nicieza A, Wootton RJ (2003) Compensatory growth in fishes: a response to growth depression. Fish Fish 4:147-190

AOAC (1984) Official methods of analysis of the association of official analytical chemists, 14th edn. Association of Official Analytical Chemists, Washington DC

Bjorndal KA, Bolten AB, Dellinger T, Delgado C, Martins HR (2003) Compensatory growth in oceanic loggerhead sea turtles: response to a stochastic environment. Ecology 84:1237-1249

Buhlmann KA, Akre TSB, Iverson JB, Karapatakis D, Mittermeier RA, Georges A, Rhodin AGJ, Van Dijk PP, Gibbons JW (2009) A global analysis of tortoise and freshwater turtle distributions with identification of priority conservation areas. Chelonian Conserv Biol 8:116-149

Chatakondi NG, Yant RD (2001) Application of compensatory growth to enhance production of channel catfish Ictalurus punctatus. J World Aquac Soc 32:278-285

Cheung SM, Dudgeon D (2006) Quantifying the Asian turtle crisis: market surveys in southern China, 2000-2003. Aquat Conserv 16:751-770

Du WG, Hu L, Lu JL, Zhu L (2007) Effects of incubation temperature on embryonic development rate, sex ratio and post-hatching growth in the Chinese three-keeled pond turtle, Chinemys reevesii. Aquaculture 272:747-753

Fermin AC (2002) Effects of alternate starvation and refeeding cycles on food consumption and compensatory growth of abalone, Haliotis asinina (Linnaeus). Aquac Res 33:197-202

Fong JJ, Parham JF, Shi HT, Stuart BL, Carter RL (2007) A genetic survey of heavily exploited, endangered turtles: caveats on the conservation value of trade animals. Anim Conserv 10:452-460

Fordhama DF, Georges A, Corey B (2007) Optimal conditions for egg storage, incubation and post-hatching growth for the freshwater turtle, Chelodina rugosa: science in support of an indigenous enterprise. Aquaculture 270:105-114

Gurney WSC, Jones W, Veitch AR, Nisbet RM (2003) Resource allocation, hyperphagia and compensatory growth in juveniles. Ecology 84:2777-2787

Hayward RS, Noltie DB, Wang N (1997) Use of compensatory growth to double hybrid sunfish growth rates. Trans Am Fish Soc 126:316-322

Hayward RS, Wang N, Noltie DB (2000) Group holding impedes compensatory growth of hybrid sunfish. Aquaculture 183:299-305

Jobling M (2010) Are compensatory growth and catch-up growth two sides of the same coin? Aquac Int 18:501-510

Jobling M, Meløy OH, dos Santos J, Christiansen B (1994) The compensatory growth response of the Atlantic cod: effects of nutritional history. Aquac Int 2:75-90

Kim MK, Lovell RT (1995) Effect of feeding regimens on compensatory weight gain and body tissue changes in channel catfish, Ictalurus punctatus in ponds. Aquaculture 135:285-293

Lu P, Wang P (1993) Effects of feeding and food deprivation on energy metabolism of juvenile Chinese three-keeled pond turtle Chinemys reevesii. J East China Normal Univ 1993:109-112

Luo Z, Tan XY, Wang WM, Fan QX (2009) Effects of long-term starvation on body weight and body composition of juvenile channel catfish, Ictalurus punctatus, with special emphasis on amino acid and fatty acid changes. J Appl Ichthy 25:184-189

Mutalib AHA, Fadzly N, Foo R (2013) Striking a balance between tradition and conservation: general perceptions and awareness level of local citizens regarding turtle conservation efforts based on age factors and gender. Ocean Coast Manage 78:56-63

Oh SY, Noh CH (2006) Effect of restricted feeding regimes on compensatory growth and body composition of red sea bream, Pagrus major. J World Aquac Soc 38:443-449

Qian X, Cui Y, Xiong B, Yang Y (2000) Compensatory growth, feed utilization and activity in gibel carp, following feed deprivation. J Fish Biol 56:228-232

Roark AM, Bjorndal KA, Bolten AB (2009) Compensatory responses to food restriction in juvenile green turtles (Chelonia mydas). Ecology 90:2524-2534 
Srijila CK, Babitha Rani AM, Girish Babu P, Tiwari VK (2014) Ration restriction, compensatory growth and pituitary growth hormone gene expression in Labeo rohita. Aquac Int 22:1703-1710

Thompson KR, Webster CD, Morgan AM, Grisby EJ (2000) Effects of different feeding frequencies on growth, body composition, and fillet composition of juvenile sunshine bass, Morone chrysops $\times$ M. saxatilis, grown indoors. J Appl Aquac 10:55-65

Tian X, Qin JG (2004) Effects of previous ration restriction on compensatory growth in barramundi Lates calcarifer. Aquaculture 235:273-283

Vonesh JR, Bolker BM (2005) Compensatory larval responses shift trade-offs associated with predator-induced hatching plasticity. Ecology 86:1580-1591

Wang J, Niu CJ, Huang CX, Rummer JL, Xie ZG (2011) Compensatory growth in juvenile freshwater turtles, Chinemys reevesii, following feed deprivation. J World Aquac Soc 42:82-89

Wei L, Zhang X, Li J, Huang G (2008) Compensatory growth of Chinese shrimp, Fenneropenaeus chinensis following hypoxic exposure. Aquac Int 16:455-470

Wilson PN, Osbourn DF (1960) Compensatory growth after undernutrition in mammals and birds. Biological Rev 35:324-361

Won ET, Borski RJ (2013) Endocrine regulation of compensatory growth in fish. Front Endocrin 4:74

Xie ZG, Niu CJ (2007) Effects of partial and complete food deprivation on compensatory growth of juvenile soft-shelled turtle (Pelodiscus sinensis): temporal patterns in growth rate and changes in body composition. Acta Hydrobiol Sinica 31:214-219

Xie QS, Yang ZC, Li JW, Li YJ (2012) Effect of protein restriction with subsequent re-alimentation on compensatory growth of juvenile soft-shelled turtles (Pelodiscus sinensis). Aquac Int 20:19-27

doi:10.1186/2193-1801-3-687

Cite this article as: Xu et al: Compensatory growth responses to food restriction in the Chinese three-keeled pond turtle, Chinemys reevesii. SpringerPlus 2014 3:687.

\section{Submit your manuscript to a SpringerOpen ${ }^{\circ}$ journal and benefit from:}

- Convenient online submission

- Rigorous peer review

- Immediate publication on acceptance

Open access: articles freely available online

- High visibility within the field

- Retaining the copyright to your article

Submit your next manuscript at $\gg$ springeropen.com 